\title{
ANALYSIS OF MODEL AND ANISOTROPY OF SURFACE WITH IRREGULAR ROUGHNESS
}

\author{
Natalija Bulaha, Janis Rudzitis \\ Riga Technical University, Latvia \\ natalija.bulaha@rtu.lv,mti@rtu.lv
}

\begin{abstract}
In this scientific work investigation of surface with irregular roughness was made with the aim to check the compliance of the surface roughness model with theory and make evaluation of roughness anisotropy, using surface roughness 3D and 2D parameters. Surface roughness measurement experiments were carried out using the measuring equipment Taylor Hobson Talysurf Intra 50. Analysis of the surface roughness model has shown that roughness ordinate distribution function complies with the normal Gaussian distribution law, which was provided by verification of a root mean square height, kurtosis and skewness of ordinate distribution function. During the roughness anisotropy assessment the problem related to the roughness spacing parameters $R S m_{1}$ and $R S m_{2}$ was identified. The analysis showed that the values of the spacing parameter $R S m_{2}$ (in direction parallel to treatment traces) are not reliable, what can be explained by divergence of surface mean plane and mean line of individual profiles, therefore determination of the surface roughness anisotropy coefficient $c$ will not be correct. In this work it was offered to use the surface texture parameter Str instead of the anisotropy coefficient $c$, what was proven experimentally - for different types of surfaces.
\end{abstract}

Keywords: surface texture, roughness anisotropy, surface model.

\section{Introduction}

Nowadays, the issue of machine part quality is closely related to the surface roughness determination and assurance. There are several methods how to accurately determine the surface roughness values. Modern measuring apparatus make it possible to get 3D roughness parameters and additional 2D parameters for individual surface profiles. Different types of mechanical treatment are used for obtaining surfaces with necessary exploitational characteristics in the field of mechanical engineering.

For machine elements and other parts, which are working in a friction pair, or for coating deposition, the mechanical treatment with abrasive instruments is a necessary operation, which will ensure a high quality of surface. After abrasive treatment the surface has an irregular roughness, which is characterised by chaotic location of microirregularities over the surface. Depending on the surface structure surface roughness can be isotropic or anisotropic. Anisotropy is typical for surfaces, treated with the grinding wheel, hon, lap and other instruments that leave treatment traces on the surface. In turn, isotropy is typical for surfaces after sandblasting, spark erosion, shot peening etc.

In order to make an analysis, calculations and implication of roughness parameters into engineering tasks it is important to check whether the surface roughness model corresponds to the theory, on the basis of which formulas for parameter calculation were created. In this scientific work a model of a surface with irregular roughness and surface anisotropy will be explored.

\section{Materials and methods}

In order to determine a model of surface with irregular roughness and compare it with the theoretical, it is necessary to perform roughness measurement experiments. Obtainment of the surface roughness parameters, topography, correlation function and ordinate distribution histogram will help define the main characteristics of the roughness model.

Seven different types of surfaces, obtained by grinding, lapping, sliding experiment and coating deposition were selected to make the experiments (see Table 1).

The measuring equipment Taylor Hobson Talysurf Intra 50 with a $16 \mathrm{~nm}$ resolution and standard stylus arm 112/2009 were used for surface roughness determination experiments [1]. The sample surface was degreased and cleaned by spirit, and then placed on the measuring table before the experiment. First of all, for each surface the profile measurements were made in order to determine the parameter $R a$ - arithmetical mean height, which is required to define the sample length and evaluation length [2] of the measuring object. Furthermore, the necessary number of measuring points was determined using the methodology, described in the literature source [3]. The next step was to enter 
certain information into Taylor Hobson software program multra: number of points and evaluation length along the $X$ and $Y$ axes, taking into account acceleration and braking particularities [4] of the measuring mechanism.

Table 1

Samples for surface roughness measurement experiment

\begin{tabular}{|c|c|}
\hline Sample number & Mechanical treatment \\
\hline $1-3$ & Surface grinding \\
\hline 4 & Cylindrical grinding \\
\hline 5 & Surface after the sliding experiment \\
\hline Sample number & Mechanical treatment \\
\hline 6 & Lapping \\
\hline 7 & Nanocoating \\
\hline
\end{tabular}

The file with surface initial topography was saved in the program Talymap expert after the experiment. In turn, to carry out an analysis of roughness parameters, the initial surface topography was filtered [5]. Surface filtration included the surface texture levelling, separation of form and waviness. 3D roughness parameters and additional 2D parameters for particular surface cross sections were determined after surface filtration. The filtered surface topographies are shown in Fig. 1.

a)

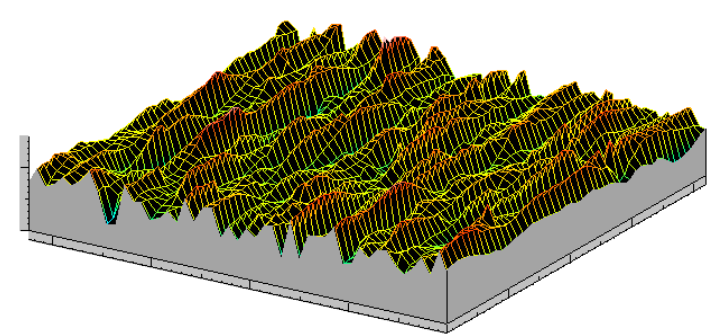

b)

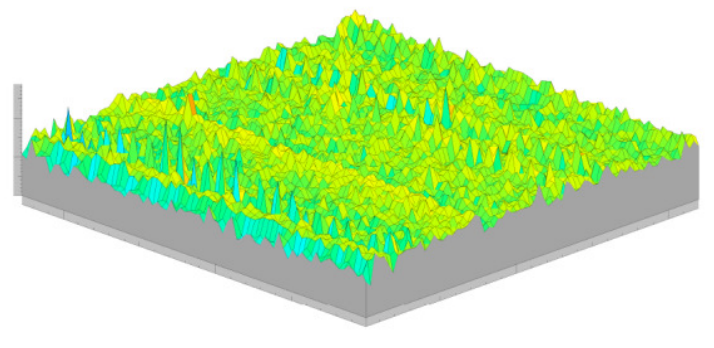

Fig. 1. Surface topography after filtration: $a$ - flat grinded surface; $b$ - surface after lapping [6]

\section{Results and discussion}

So, it is important to define the model components to make an analysis of the surface roughness model. In general surface roughness is described by two functions: surface ordinate distribution and correlation function [7].

The first step was the evaluation of the roughness ordinate distribution. Theoretically, the ordinate distribution function for surface with irregular roughness must conform to normal Gaussian distribution law, where the probability that the surface ordinate values will be clustered in the interval from $-3 \sigma$ to $+3 \sigma$ is equal to $99.7 \%$, where $\sigma$-root mean square of the distribution function.

The Normal distribution law is characterized by:

- Symmetric placement of random variable in relation to the mean value of random variable;

- The following parameter values:

Table 2

Characterizers of normal distribution law

\begin{tabular}{|c|c|c|}
\hline Parameters & Name of parameter & Value \\
\hline$S k u$ & Kurtosis & 3 \\
\hline$S s k$ & Skewness & 0 \\
\hline $6 \sigma$ & Root mean square deviation & $99.7 \%$ \\
\hline
\end{tabular}

The surface roughness parameter $S k u$ shows the sharpness of the surface ordinate distribution function. If the surface microirregularities are located very close to each other, then the distribution function will be outstretched and the values of the parameter $S k u$ will be less than 3. In turn, if the surface microirregularities are not distributed densely over the surface, but at a certain distance from each other, then the distribution function will be sharp and the $S k u$ value will be greater than 3 . 
The surface roughness parameter Ssk characterizes the skewness of the ordinate distribution function. This parameter value will be negative for surfaces with deep spicy valleys (for example, plateau surfaces - honed) and positive - for surfaces with high spicy peaks. Respectively, the mean value of surface ordinates will be shifted to the right or to the left from the distribution function center (see. Fig. 2).
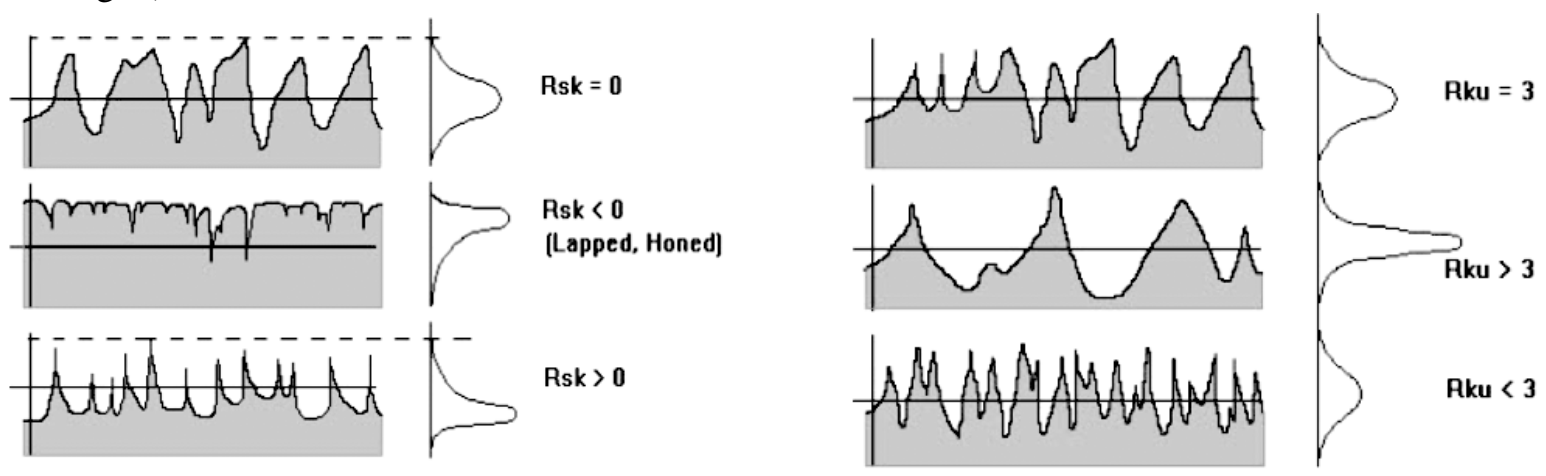

Fig. 2. Distribution functions of surface ordinates depending on values of skewness (Ssk) and kurtosis (Sku) [8]

In Fig. 3 we can see ordinate distribution histograms of three investigated surfaces. After visual assessment it can be concluded that the function of the surface ordinate distribution has an asymmetry - offset of the average value from the center.

a)

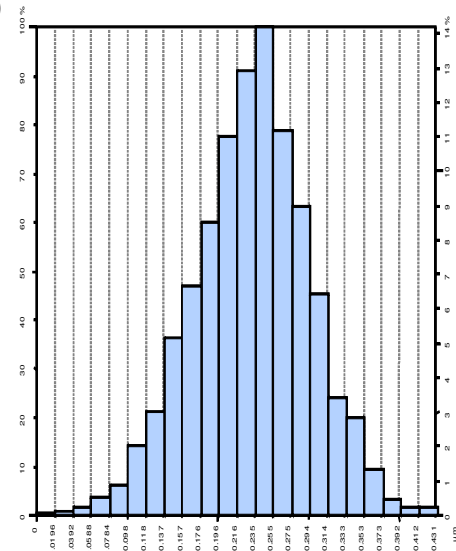

b)

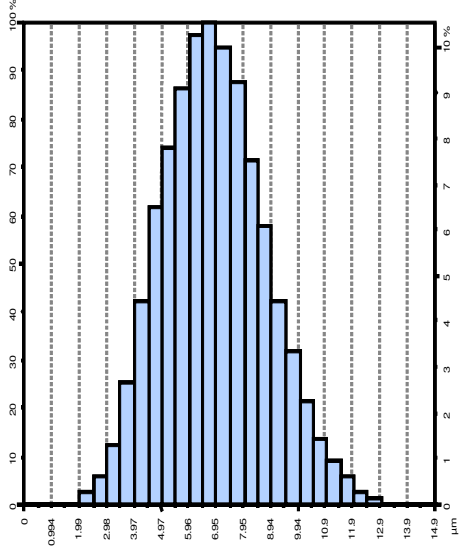

c)

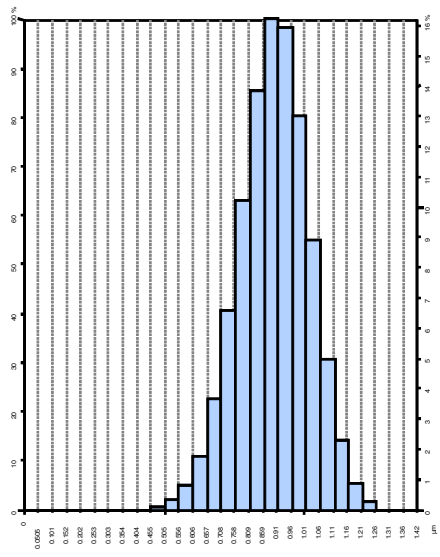

Fig. 3. Ordinate distribution histograms of surfaces with irregular roughness:

$\mathrm{a}, \mathrm{b}$ - for flat grinded surfaces; $\mathrm{c}$ - for nanocoating

Then the values of the parameters Ssk and $S k u$ were analysed using a graphical method for 7 investigated surfaces. Distribution of the parameters values is given in Fig. 4.

From Fig. 4 it can be concluded that any of the investigated surfaces have $S s k$ and $S k u$ values, which would precisely conform to the normal distribution law, because the greater part of these values does not fall within the deviation zone $\pm 10 \%$.

In addition, a $6 \sigma$ test was made determining whether the surface ordinate values are located in the interval from $-3 \sigma$ to $+3 \sigma$, what constitutes $99.7 \%$. For this purpose, several calculations were made determining $6 S q(S q=\sigma)$ value (see. Table 3 ):

Table 3

Test of normal distribution law using a $6 \sigma$ method

\begin{tabular}{|c|c|c|c|c|c|c|c|}
\hline Parameter & No.1 & No.2 & No.3 & No.4 & No.5 & No.6 & No.7 \\
\hline$S q$ & 0.062 & 1.880 & 3.970 & 4.240 & 0.097 & 0.170 & 0.125 \\
\hline $6 S q(99.7 \%)$ & 0.372 & 11.280 & 23.820 & 25.440 & 0.584 & 1.020 & 0.750 \\
\hline $100 \%$ & 0.373 & 11.314 & 23.892 & 25.517 & 0.586 & 1.023 & 0.752 \\
\hline$S t$ & 0.431 & 14.900 & 27.600 & 26.400 & 0.992 & 1.830 & 1.420 \\
\hline
\end{tabular}


The test according to Table 2 points that the ordinate values lie outside the border of $\pm 3 \sigma$. It can be concluded that was made ordinate distribution function of the surface with irregular roughness is very close to normal, but it does not conform to it completely. First of all, it can be explained by the fact that each surface is described only by one topographical map. Secondly, the real function of was made surface ordinates distribution can have deviations from the normal distribution law.

a)

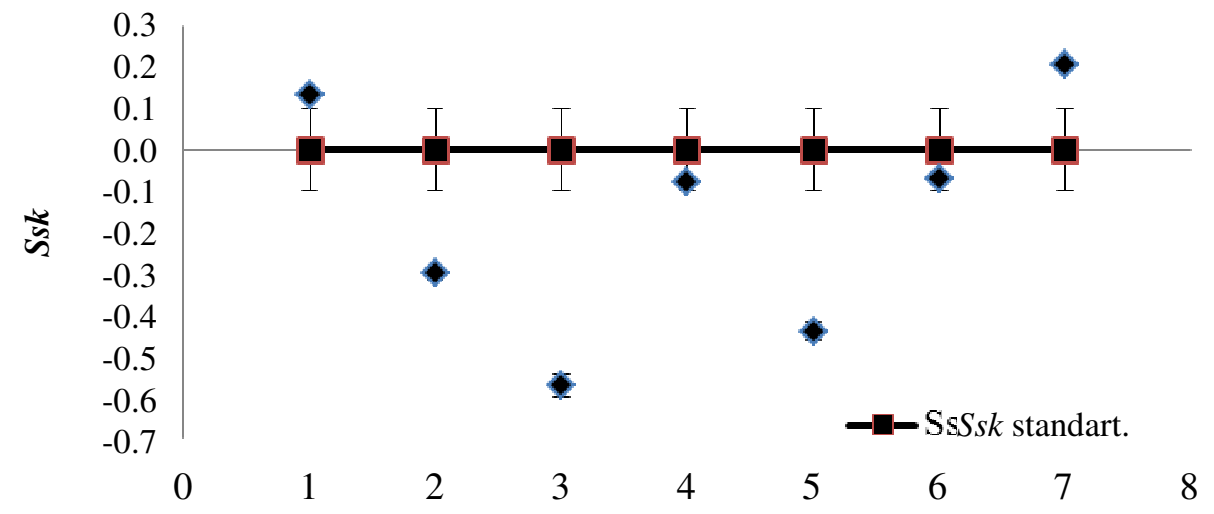

b)

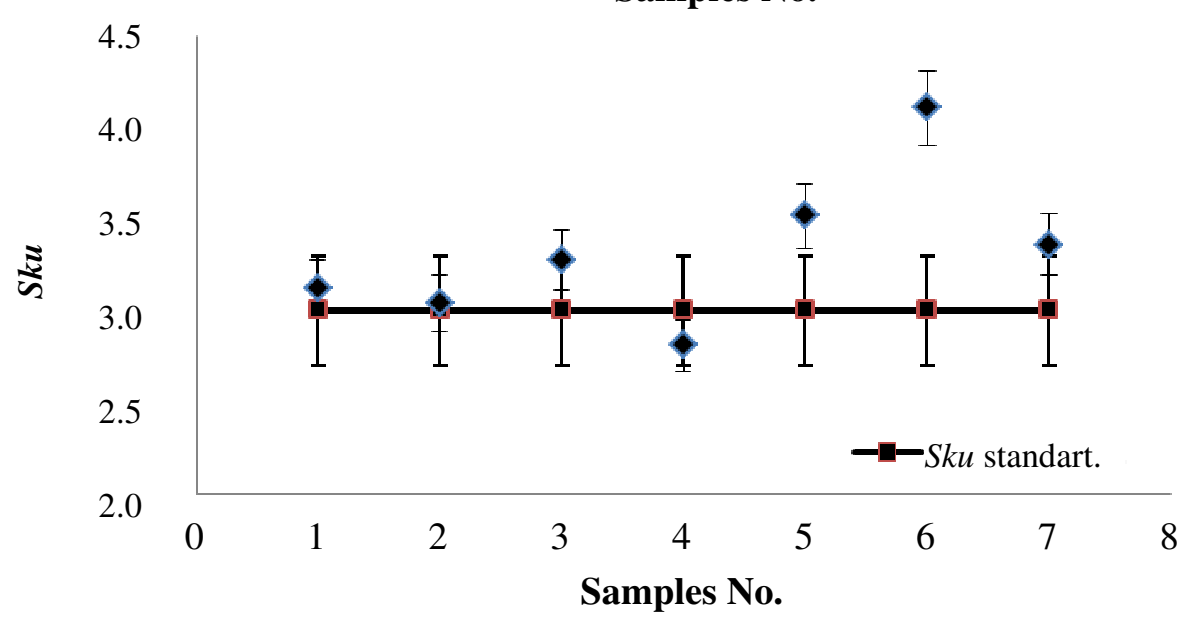

Fig. 4. Comparison of parameters $S s k$ (a) and $S k u$ (b) with normal Gaussian distribution law

The second step was evaluation of the surface roughness correlation function. As it is known, the correlation function represents the link between surface points, them moving away from each other. The correlation function is decreasing for surfaces with irregular roughness, what indicates that the link between the points is lost rather quickly. With a distance increase, a link between initial and shifted points of surface profilogramm will weaken, and correlation between two points will be lost at certain shift [7].

Principally, irregular surface is characterized by the height parameter $S q$ - root mean square height (or $S a$ - the arithmetical mean height) and $R S m_{1}, R S m_{2}$ - average steps of surface roughness along the $X$ and $Y$ axes. In general, the equation of correlation function can be expressed in the following form [7]:

$$
K(\tau)=\sigma^{2} \cdot \rho(\tau),
$$

where $(\rho) \tau$-standardized correlation function, which values change from 0 to 1 ; $\sigma-$ root mean square deviation, $\mu \mathrm{m}$.

The experimental results show that the correlation function of surfaces with irregular roughness can be described by the following equations [7]: 


$$
\rho(\tau)=\frac{1}{1+\alpha \cdot \tau^{2}},
$$

where $\alpha$-approximation parameter

$$
\rho(\tau)=\frac{\cos (\omega \tau)}{1+\alpha \cdot \tau^{2}},
$$

where $\omega$-approximation parameter.

If the correlation function is descending monotonically according to equation (2), it means that it depends only on two parameters $R \mathrm{Sm}_{1}$ and $R S m_{2}$, but if the correlation function is descending with damped oscillations according to equation (3), it is necessary to take into account the local average step $S_{1}$ and $S_{2}$ of roughness profiles [7].

In this work correlation functions for the investigated surfaces were determined and some of them are shown in Fig. 5. The correlation functions were truncated in the middle to be clearly visible their character along the $X$ and $Y$ axes. The correlation function is descending with fluctuations for all surfaces in the direction perpendicular to the processing traces, as evidenced by the small roughness step in comparison with the perpendicular direction. In turn, the correlation function is descending monotonically in the direction parallel to processing traces only for those samples, which have an expressed anisotropy; it is all grinded surfaces, surface after sliding experiment, lapping and nanocoating. If the surface structure is approaching to isotropic structure, what is characterised by similar properties in the $X$ and $Y$ axes directions, then the correlation function will have a descending type with damped oscillations in both directions.
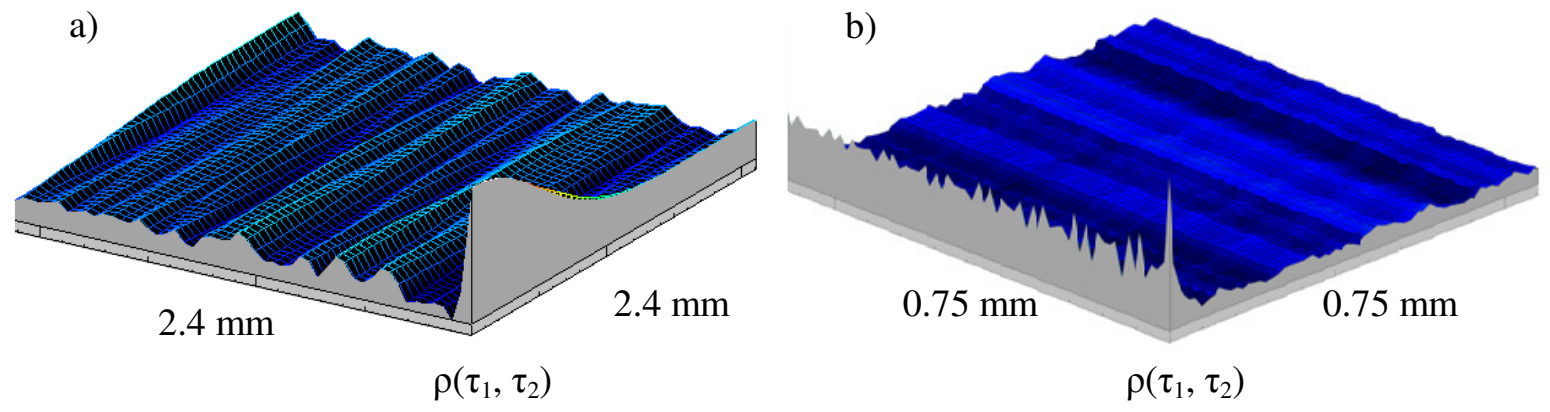

Fig. 5. Correlation function of surface roughness:

$a$ - for flat grinded surface; $b$ - for lapped surface

Since the specified type of the correlation function has a low influence on the correlation interval $\tau_{\mathrm{k}}$, where [7]:

$$
\tau_{k}=\int_{0}^{\infty}|\rho(\tau)| d t,
$$

then it can be assumed that we can rely on the monotonically decreasing nature of the correlation function in calculations for surfaces with irregular roughness.

In this scientific work additional interest was paid to the rough surface anisotropy, which is determined by the ratio of roughness steps $R S m_{1}$ and $R S m_{2}$. Anisotropic surfaces have diversity of profiles in two mutually perpendicular directions. It is peculiar for grinded surfaces, where the average step in the $\mathrm{X}$-axis direction can be 3 times smaller than in the Y-axis direction. In turn, if the ratio of mean spacing of profile irregularities approaches to 1, one can speak about surface isotropy, which is peculiar, for example, to surfaces after sandblasting. Several scientists [7] denote the ratio of surface average steps in two mutually perpendicular directions with the anisotropy coefficient $c$ :

$$
c=R S m_{1} / R S m_{2}
$$


Despite the fact that the ratio of the parameters $R S m_{1}$ and $R S m_{2}$ has not been specified in standards, it is a very important parameter, which is required for surface anisotropy description.

While making the surface roughness measurement experiments the problem related to the inaccuracy of roughness average step value in the $Y$-axis direction (parallel to processing traces) was discovered. Fig. 6 shows the surface topography and mean spacing of profile elements in the $X$ and $Y$-axis directions. For a given surface the parameter $R S m_{1}$ (perpendicular to the processing traces) is $0.13 \mathrm{~mm}$, but the parameter $R \mathrm{Sm}_{2}$ (parallel to processing traces) is $0.15 \mathrm{~mm}$, which is not possible even after the visual assessment. It turns out that the problem is connected to the divergence of the surface mean plane and the profile mean line. Normally, the mean plane of the surface must match the mean line of the profile, but actually, only in the $\mathrm{X}$-axis direction the mean line of the profile is located at the same level with the mean plane, what has been determined in literature source [9].

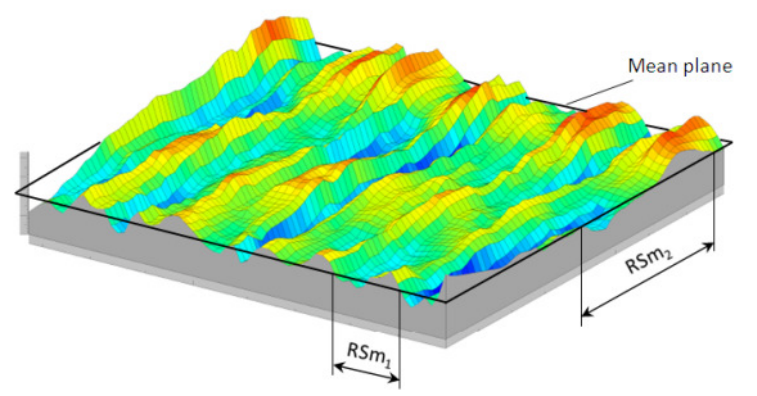

Fig. 6. Alignment of 3D surface roughness steps [9]

So, making the surface roughness measuring experiments, it is not possible to obtain reliable values of the parameter $\mathrm{RSm}_{2}$, and respectively to calculate the anisotropy coefficient $c$. In this work it was offered to use the surface texture parameter Str from the Standard ISO 25178-2 instead of the anisotropy coefficient $c$. Texture aspect ratio $S t r$ is defined as the ratio of the horizontal distance of the autocorrelation $f_{A C F}(t x, t y)$, which has the fastest decay to a specified value $s$ to the horizontal distance of the $f_{A C F}(t x, t y)$, which has the slowest decay to $s$, with $0 \leq s<1$ [10]. It means that this parameter depends on the decrease of the surface correlation function. As it was mentioned above, one of the correlation function descriptors is the surface roughness average step, so it could be argued that there is a relationship between the parameters $S t r$ and $R S m_{1}, R S m_{2}$.

On the basis of the relationship from the literature source [6], which expresses that at definitelyoriented correlation function (where its correlation intervals $\tau_{k x}$, $\tau_{k y}$ coincide with the $X$ and $Y$ axis directions) it is possible to make a comparison between the anisotropy coefficient $c$ and the parameter Str, it can be stated:

$$
\operatorname{Str}=\frac{\tau_{k x}}{\tau_{k y}}
$$

where $\tau_{k x}-$ correlation interval along the direction $X$;

$\tau_{k y}$ - correlation interval along the direction $Y$.

Since we did not know the reliable values of the roughness average step $R S m_{2}$, the anisotropy coefficient $c$ (in further text $-c_{v i z}$ ) was determined visually, using the methodology described in literature source [9]. The relationship between $c_{v i z}$ and the texture aspect ratio Str was modelled graphically (see Fig. 7).

In Fig. 7 it is shown that there is a strong correlation between the texture aspect ratio and the anisotropy coefficient, what is indicated by the high value of the coefficient of determination $R^{2}$, which expresses the relationship between the variables. According to the obtained results it can be concluded that the texture parameter Str can be applied for determination of surface roughness anisotropy and the mean spacing of profile irregularities $R s m_{2}$ in the direction parallel to processing traces. 


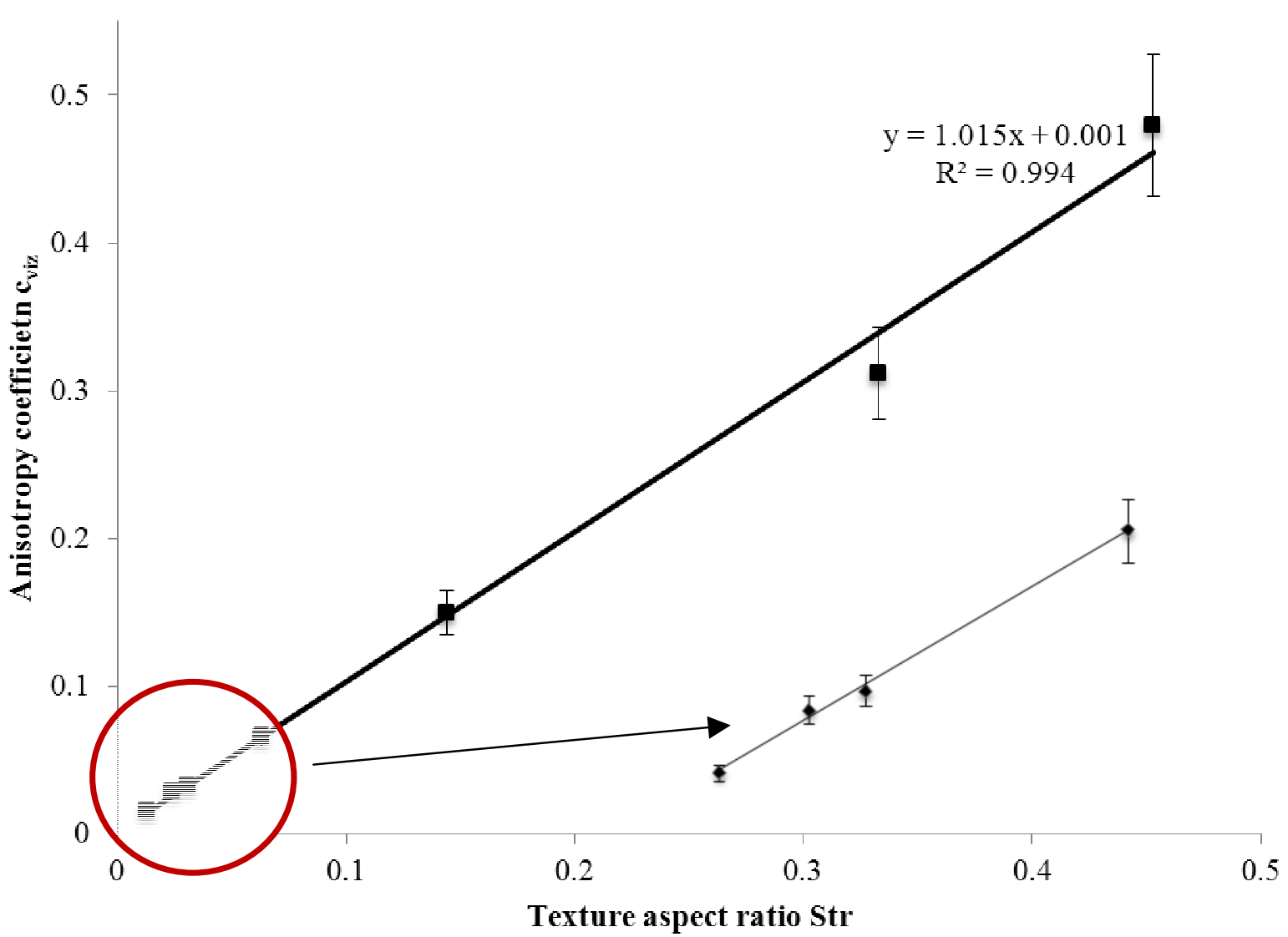

Fig. 7. Relationship between anisotropy coefficient $c_{v i z}$ and texture aspect ratio $\mathrm{Str}$

\section{Conclusions}

1. Ordinate distribution function for surfaces with irregular roughness is very close to normal Gaussian distribution low, what has been proven by determination of kurtosis, skewness and $6 \sigma$.

2. Correlation functions for surfaces with irregular roughness have decreasing nature, what indicates that there is a weak link between the surface points moving them away from each other. In addition for surfaces with expressed anisotropy in the direction parallel to processing traces the correlation function will decrease monotonically, but in the perpendicular direction - with damped oscillations.

3. The obtained surface roughness model provides calculations of roughness parameters using random field equations.

4. For determination of the surface roughness anisotropy parameter Str the standard ISO 25178-2 can be applied.

\section{References}

1. Form Talysurf Intra. [online] [01.03.2016], Available at: http://www.taylor-hobson.com/uploads/downloads/products/FTS \%20Intra \%20brochure_ Lowres_EN.pdf.

2. LVS EN ISO 4288 standard "Geometrical Product Specifications (GPS) - Surface texture: Profile method - Rules and procedures for the assessment of surface texture".

3. Bulaha N., Rudzitis J., Lungevics J., Cudinovs V. Measurement principles of 3D roughness parameters. Proceedings of International conference "Engineering for Rural Development 2016", May 25-27, 2016, Jelgava, Latvia, pp. 1059-1064.

4. Stout K.J., Sullivan P.J., Dong W.P., Mainsah E., Mathia N.T., Zahouani H. Development of Methods for Characterization of Roughness in Three Dimensions, First edition. London: Penton Press, 2000, 384 p. 
5. Exploring Surface Texture, 7th edition. Great Britain: Taylor Hobson Limited, 2011, 110 p., [online] [01.03.2016], Available at: http://www.taylor-hobson.com/uploads/learningzone/ metrology-books/Exploring \%20Surface \%20Texture \%202014.pdf.

6. Bulaha N., Rudzitis J., Lungevics J., Linins O., Krizbergs J. Research of surface roughness anisotropy Latvian journal of physics and technical sciences, vol.54, 2017 (submitted to publication).

7. Рудзитис Я.А. Контактная механика поверхностей. Часть 1. (Mechanics of surface contact. Part 1). Riga: Riga Technical University, 2007. 193 p. (in Russian).

8. Bitelli G., Simone A., Girardi F., Lantieri C. Laser scanning on road pavements: a new approach for characterizing surface texture. Sensors, vol.12, 2012, pp. 9110-9128.

9. Bulaha N., Rudzitis J., Lungevics J., Linins O., Berzins K. Analysis and calculation of spacing parameters of anisotropic 3D surface roughness. Latvian journal of physics and technical sciences, vol.54, 2017 (submitted to publication).

10. LVS EN ISO 25178-2 standard "Geometrical product specifications (GPS) - Surface texture: Areal - Part 2: Terms, definitions and surface texture parameters". 